Full Paper

\title{
Kaliumverfügbarkeit im Boden, Kaliumaufnahme und die Auswirkungen auf die pH-Werte der Moste und Weine
}

\author{
Potassium availability in soil, potassium uptake and effects on the $\mathrm{pH}$ of musts and wines \\ Disponibilità di potassio nel suolo, assimilazione del potassio ed effetti sul pH dei mosti e vini
}

${ }^{1}$ Versuchzentrum Laimburg

\section{ABSTRACT}

The $\mathrm{pH}$ values of South Tyrolean musts and wines are high in most years. The high $\mathrm{pH}$ values are not only related to the low content of organic acids of the grapes at maturity, they are also the result of high potassium levels in the must. It should therefore be examined whether vineyards with low potassium uptake would produce musts with lower $\mathrm{pH}$-values and if it would be appropriate to focus even more on a targeted and possibly even lower potassium availability for the vines.

For this purpose, three sites, which repeatedly showed low levels of potassium in the leaves in recent years, and three better supplied sites have been studied for potassium levels in the soil, in the leaf and from the musts to the finished wine.

It turned out that all sites, even the moderately supplied ones, still had too high potassium contents in the soils to cause significantly lower potassium contents in the musts. Even in years with very low potassium levels in the leaf during flowering and early ripening, more than $1.5 \mathrm{~g} / \mathrm{l}$ of potassium were still found in the musts. In pronounced periods of drought, which have been more frequent in recent years as a result of climate change, the potassium uptake by the vine is severely limited as long as the drought persists, but the deficiencies can be quickly compensated for as soon as higher soil moisture is present.

\section{KEYWORDS}

Grapevine, soil potassium, leaf potassium, must potassium, $\mathrm{pH}$ value

\section{CITE ARTICLE AS}

Raifer Barbara, Haas Florian Stanek Katharina et.al. (2019) Potassium availability in soil, potassium uptake and effects on the $\mathrm{pH}$ of musts and wines. Laimburg Journal 1/2019 DOI: $\underline{10.23796 / \text { LJ/2019.003 }}$

\section{CORRESPONDING AUTHOR}

Barbara Raifer

Laimburg 6, Pfatten, I-39040 Auer

(BZ), Italien

barbara.raifer@laimburg.it

+390471969616 


\section{EINLEITUNG}

Die ansteigenden Temperaturen infolge der Klimaänderung führen insgesamt zu einem höheren Reifegrad der Trauben zur Ernte, das bedingt höhere Zucker-, aber niedrigere Säuregehalte in den Mosten und alkoholreichere aber zugleich säureärmere Weine. In Südtirol war von 1920 bis 2010, wie auch in anderen Regionen des Alpenraumes, ein stärkerer Temperaturanstieg als im globalen Mittel zu verzeichnen (Adler et al., 2015) [1]. Das hat dazu geführt, dass das Anreichern der Moste und Weine mit Säure heute eine verbreitete Kellerpraxis ist. Der Rolle des Kaliums in diesem Zusammenhang wird dabei meist wenig Beachtung geschenkt.

Die Wahrnehmung des sauren Geschmackes hängt nicht allein von den verschiedenen im Most und Wein vorkommenden Säuren ab. Vielmehr bestimmt der pH-Wert, also die Konzentration der Wasserstoff-Ionen, wie sauer der Most oder Wein empfunden wird. Neben der Konzentration der einzelnen Säuren wirkt sich der Gehalt an gelöstem Kalium auf den pH-Wert aus (Boulton, 1980) [2]. Während höhere Säuregehalte den $\mathrm{pH}$-Wert senken, steigt dieser mit zunehmenden Kaliumgehalten an. Bei hoher Kaliumaufnahme und Einlagerung in die Beeren verbindet sich nämlich ein Teil des Kaliums mit den Säuren, und Weinsäure fällt als Kaliumhydrogentartrat, dem sogenannten „Weinstein“, aus.

Daher ist eine mäßige Kaliumversorgung, die darauf abzielt, den Kalium-Bedarf der Rebe zu decken, aber jeden Luxuskonsum zu vermeiden, sinnvoll und erstrebenswert. Sie kann dazu beitragen, trotz der heutigen, oft eher niedrigen Säurewerte zur Ernte, den $\mathrm{pH}$-Wert der Moste und Weine in einem angemessenen Bereich zu bewahren und die Notwendigkeit der Zugabe von Säure zu erübrigen oder zu begrenzen.

Wie deutlich sich eine unterschiedliche Kaliumversorgung auf den $\mathrm{pH}$-Wert der Moste und Weine auswirken kann, zeigte Ryser et al., 1989 [3], anschaulich auf. Offensichtlich nehmen Reben bei reichlicher Kaliumversorgung der Böden deutlich mehr Kalium auf als sie benötigen, und ein Teil dieses „überzähligen“ Kaliums gelangt in die Beeren, bzw. in die Moste und Weine.

Ein hoher Kaliumgehalt führt zu vollen, weichen bis hin zu unharmonischen, breiten und seifigen Weinen (Schneider, 1998) [4]. Bei sehr niedrigen Kaliumgehalten sind die Säuren hingegen schlecht gepuffert und die Weine wirken schlank und spitz.
Auch für das Wachstum der Rebe ist weder ein zu viel noch ein zu wenig an Kalium erstrebenswert. Ein hoher Kaliumgehalt verschlechtert die Aufnahme von Magnesium und trägt zum Auftreten von Stiellähme (Keller, 2015) [5] und vermutlich auch zur Entwicklung der „Grünen Beeren“ bei der Sorte Gewürztraminer bei. Ein Defizit an Kalium in der Bodenlösung hingegen wirkt sich ungünstig auf die Entwicklung der Rebe aus, zunächst auf das Wurzelwachstum, dann auf die vegetative Entwicklung, führt zu erhöhter Trockenstressanfälligkeit und mindert die Winterfestigkeit der Reben. Bei fortgeschrittenem Mangel wird die Zuckereinlagerung in die Beeren gehemmt.

In einigen Weinbaugebieten kommen „kaliumfixierende" Böden vor. Sie halten Kalium in den Schichtzwischenräumen von Tonmineralen fest. Derartige Böden treten im Südtiroler Weinbaugebiet nicht auf. Kaliummangelsituationen sind im Südtiroler Weinbau so gut wie unbekannt und beschränken sich auf Neuanlagen, welche auf zuvor als Futterwiesen und Weiden genutzten Standorten angelegt wurden. Zur guten Kaliumversorgung der Südtiroler Weinbauböden dürfte auch die jahrzehntelang verbreitete Dauerbegrünung mit beitragen. Die Wurzeln der Begrünungspflanzen entnehmen dem Boden unter anderem auch beachtliche Mengen an Kalium, welches nach dem Mulchen oder Mähen, bei der Verrottung der Grünmasse, wieder freigesetzt wird. Diese Art der Bodenbewirtschaftung trägt daher dazu bei, Kalium im Oberboden anzureichern.

Es ist bekannt, dass die Kaliumaufnahme mit steigendem Wassergehalt der Böden deutlich zunimmt, während in Trockenphasen mit hoher Bodenaustrocknung die Kaliumaufnahme eingeschränkt ist (Hepner et al., 1985) [6].

In den letzten zwei, drei Jahrzehnten wurde die Kaliumdüngung im Südtiroler Weinbau deutlich reduziert, teilweise über Jahre ausgesetzt. Dies aufgrund der oft eher hohen Kaliumgehalte laut den Blatt- und Bodenanalysen und den heutigen, wesentlich geringeren Ertragsmengen, welche den Böden auch entsprechend niedrigere Kaliummengen entziehen. Besonders in den Trockenjahren werden aber inzwischen teilweise sehr niedrige Kaliumwerte bei Blattanalysen gefunden. Ziel der vorliegenden Arbeit war es daher, erste Einblicke zu gewinnen, ob einzelne Rebanlagen sich Kalium-Mangelsituationen nähern oder teilweise bereits im Mangelbereich sind, und wie sich dies auf die Kaliumeinlagerung in die Beeren und auf die $\mathrm{pH}$-Werte der Moste/Weine auswirkt. Im Jahre 2015 wurde die vorliegende Arbeit im Rahmen einer Diplomarbeit begonnen.

\section{MATERIAL UND METHODEN}

\section{VERSUCHSFLÄCHEN}

Die sechs untersuchten Standorte gehören zum Einzugsgebiet der Kellerei Tramin. Laut Blattanalysen der vorangegangenen Jahre waren die Kaliumwerte in diesen Anlagen zum Teil sehr niedrig. Alle sechs Weingärten befinden sich in der Gemeinde Tramin, nördlich von Schloss Rechtenthal und südlich vom Ortskern von Söll und liegen somit räumlich nahe beisammen. Zur Auswahl der Flächen wurde im April 2015 eine Bodenuntersuchung durchgeführt, wobei aufgrund der starken Bodenaustrocknung zu diesem Zeitpunkt nur der Oberboden bis $30 \mathrm{~cm}$ Tiefe beprobt werden konnte. Aus 11 beprobten Anlagen wurden schließlich die drei Flächen, deren Analyse die geringsten (11, 16, 20 mg Kalium pro 100 g Boden) und jene drei Flächen deren Analyse die höchsten (31, 33, 38 mg Kalium pro 100 g Boden) Kaliumgehalte aufwiesen, ausgewählt. In ihren physikalischen und teilweise auch chemischen Eigenschaften waren die Böden einander ähnlich. Bei den drei südlicher gelegenen Anlagen handelte es sich um lehmige Sandböden, gegen Söll hingegen weisen die Böden geringere Sandanteile auf: bei zwei Anlagen handelte es sich um sandige Lehme und die nördlichste Anlage wies einen schluffigen Lehmboden auf. Gemein ist den Böden ein $\mathrm{pH}$-Wert, der sich zwischen 7.0 und 7.3 bewegt.

Die Anlagen wurden von den Besitzern betriebsüblich bewirtschaftet. 3 Anlagen wiesen eine Herbsteinsaat auf, die anderen drei waren dauerbegrünt. Alle Anlagen wurden in der Zeit von April bis September zwei bis drei Mal gemulcht. In den beiden Erhebungsjahren fand keine Düngung statt.

Alle Anlagen waren mit der Sorte Gewürztraminer auf der Unterlage $\mathrm{SO} 4$ bepflanzt und zu Versuchsbeginn zwischen 8 und 12 Jahre alt. Alle Anlagen waren als Spalier mit Flachbogen erzogen, der Standraum pro Rebe variierte von $1.36-1.60 \mathrm{~m}^{2}$.

\section{ANALYSEN}

Im Laufe des Versuchs wurden Böden, Blätter und Beeren, Moste und Weine beprobt. 
Für die Beprobung der Böden wurde für jede Einzelprobe mehrmals mit dem Erdbohrer aus der entsprechenden Bodenschicht (Oberboden 0-30 cm, Unterboden 30-60 cm Tiefe) im Unterstockbereich Boden entnommen.

Zur Bodenuntersuchung wurden die Böden im Labor des Versuchszentrums Laimburg getrocknet, auf $2 \mathrm{~mm}$ Korngröße gesiebt, dann erfolgte die taktile Bestimmung der Bodenart und weiter wurden folgende Analysen durchgeführt: Organischer Kohlenstoff ausgedrückt als Humus nach ISO 10694:1995, Phosphor in CAL-Extrakt ausgedrückt als P2O5, Kalium in CAL-Extrakt ausgedrückt als K2O nach ÖNORM L 1087:2012 A.5, pH in $\mathrm{CaCl} 2$ nach DIN EN 15933:2012, Magnesium und Bor, sowie der Carbonatgehalt.

Blattproben erfolgten zu drei bis vier Terminen (Vollblüte, Reifebeginn, Weichwerden und Ernte). Die Blätter wurden aus der Traubenzone entnommen, soweit möglich jene, welche den Gescheinen/Trauben gegenüberlagen. Die Blätter wurden im Labor für Boden- und Pflanzenanalysen des Versuchszentrums Laimburg gewaschen, getrocknet, gemahlen und auf folgende Elemente untersucht: Stickstoff nach DIN EN ISO 166341:2009, Phosphor, Kalium, Calcium, Magnesium, Bor, Eisen, Mangan, Kupfer und Zink nach EPA 30521996 + EPA 6010D 2014

Reifetests: Grundsätzlich wurden pro Traube je drei Beeren entnommen - eine im oberen, eine im mittleren und eine im unteren Bereich der Traube. Die Reifetests fanden zu drei Terminen statt, zwei erfolgten im Laufe der Reifephase, der letzte zur Lese. Beide Wiederholungen der sechs Weingärten wurden getrennt beprobt und untersucht. Die Beeren wurden bei der Entnahme gezählt, gewogen, gepresst $(0.1,0.2$ und 0.3 bar für jeweils $20 \mathrm{sec}$.), zentrifugiert (zwischen 3000 und $9000 \mathrm{rpm}$ ), filtriert auf $<5 \mu \mathrm{m}$ und mittels FTIR (FOSS ${ }^{\circledR}$ ) auf folgende Inhaltsstoffe analysiert: Mostgewicht, ${ }^{\circ} \mathrm{KMW}$, pH-Wert, Gesamtsäure, hefeverfügbaren Stickstoff, Gehalt an Aminogruppen, Gehalt an Ammonium, Apfelsäure, Weinsäure, Gluconsäure und Kalium. Kalium wurde zusätzlich zur Messung mittels FTIR auch enzymatisch bestimmt.

\section{ERTRAG}

Der Ertrag der einzelnen Versuchsanlagen wurde aus der Gesamternte der Anlage und der Netto-Grundstücksgröße errechnet. Der Ertrag der nicht ausgedünnten Parzelle „Tal“ im Jahre 2016 wurde durch Zählen der Trauben pro Stock und der ermittelten durchschnittlichen Traubengröße errechnet.

\section{NANOVINIFIKATION}

Bei erreichter Reife der Trauben wurden je Anlage und Wiederholung rund $2 \mathrm{~kg}$ Trauben geerntet, indem aus den Trauben einzelne Teile aus dem oberen, mittleren und unteren Traubenbereich herausgeschnitten wurden. Die Trauben wurden zügig verarbeitet, gepresst und die Moste wurden kühl gestellt. Am darauffolgenden Tag wurden die Moste vom Trub abgezogen und mit 0.3 g ymaflore ${ }^{\circledast}$ VL-2 Hefen/L versetzt und bei $20^{\circ} \mathrm{C}$ vergoren.

Bei Gärende (weniger als 2 g Restzucker) wurden die Weine von der Hefe abgezogen und ins Weinlabor zur Analyse gebracht. Die Vorbereitung für die FOSS ${ }^{\circledR}$ Analyse (FTIR) erfolgte nach dem gleichen Ablauf wie bei den Reifetests. Untersucht wurden: Alkoholgehalt, pH-Wert, Gesamtsäure, Apfelsäure, Weinsäure, Gluconsäure, Milchsäure, Zitronensäure, Glyzerin, Extrakt, Flüchtige Säure und Kalium. Anschließend kamen die Weine drei Wochen lang zur Kältestabilisierung bei $4{ }^{\circ} \mathrm{C}$ in den Kühlschrank. Nachfolgend wurden die Weinanalysen mittels FTIR und Kalium, zusätzlich auch enzymatisch, wiederholt.

\section{DATENANALYSE}

Für die statistische Analyse mittels Anova und bivariater Korrelation wurde IBM ${ }^{\circledR}$ SPSS Statistics herangezogen.

\section{ERGEBNISSE}

\section{WETTERDATEN IN DEN ZWEI VEGE- TATIONSPERIODEN}

Kalium kann bei guter Bodenfeuchte von der Rebe wesentlich leichter aufgenommen werden als bei fortgeschrittener Bodenaustrocknung (Hepner et al., 1985) [6], deshalb spielen die Feuchtigkeitsverhältnisse in den beiden Untersuchungsjahren für die Interpretation der Ergebnisse eine Rolle. In Abbildung 1 sind die Monatsniederschläge der Jahre 2015 und 2016 wiedergegeben. Die Messwerte stammen von der Wetterstation Laimburg, wurden also einige Kilometer von den Untersuchungsstandorten entfernt ermittelt und zeigen somit auch nur die allgemeine Niederschlagssituation der beiden Jahre auf. Zwischen den untersuchten Standorten dürfte es kaum größere Abwei- chungen in den Niederschlägen gegeben haben, da die Standorte nahe beisammen liegen und gleich exponiert sind.

Im Jahre 2015 waren die ersten Monate des Jahres extrem trocken. Die Bodenentnahme für eine erste Bodenanalyse im April 2015 war daher auch nur im Oberboden möglich. Aufgrund des sehr trockenen und harten Unterbodens war es unmöglich, eine rapräsentative Bodenprobe zu entnehmen. Infolge der Mai-Niederschläge entspannte sich die Situation etwas, das Wasserdefizit des Bodens blieb aber bestehen. Erst im August, September und Oktober fielen intensivere Niederschläge und am 18. August 2015 war es erstmals in diesem Jahr möglich, auch aus dem Unterboden Proben für die Bodenanalyse zu entnehmen.

Im Jahre 2016 waren die Niederschläge besser verteilt. Bereits im Frühjahr war es weniger trocken und in den Monate Mai, Juni und Juli fiel ausreichend Regen.

\section{KALIUMANALYSEN DES BODENS UND DER BLÄTTER}

Am 24. April 2015 konnte nur der Oberboden für eine Analyse entnommen werden, die erste Untersuchung von Ober- und Unterboden erfolgte am 18.08.2015 (Abb. 2). Entsprechend den Ergebnissen vom 18.08.2015 und unter Berücksichtigung der Bodenart fielen die Anlagen "Schloss" und „Söll“ in die mittlere Versorgungsklasse, die Anlage „Burg" in die mittel-hohe und die restlichen drei Anlagen in die hohe bis sehr hohe Versorgungsklasse. Keine Anlage war in einem deutlichen Defizitbereich, nur die Anlage "Schloss" näherte sich diesem bei zwei Terminen.

Da die Ergebnisse der Kaliumwerte, nicht aber jene der anderen Mineralstoffe, zwischen dem ersten und zweiten Probetermin zum Teil deutlich variierten, wurde zu Saisonsende des ersten Untersuchungsjahres eine weitere Bodenanalyse entnommen. Nun fielen die Kaliumwerte durchwegs etwas tiefer aus. Besonders auffällig war der Abfall des Kaliumgehaltes der Anlagen „Berg“ und „Tal“ im Jahre 2015 vom ersten auf den zweiten bzw. auf den 3. Beprobungstermin. Derselbe Trend zeigte sich auch in den Blattanalysen des Jahres 2015 (Abb.3). Die beiden Anlagen hatten zur Blüte die höchsten Kaliumwerte der sechs Anlagen, diese fielen aber bereits zum Termin Reifebeginn deutlich ab. Mit Ausnahme der Werte der beiden Anlagen "Berg" und "Tal", zum Termin Blüte, lagen alle weiteren Blatt- 
Kaliumwerte im Jahre 2015 im Mangelbereich, wiesen also deutlich unter $1 \%$ Kalium in der Trockensubstanz auf. 2016 hingegen lagen die ermittelten Werte der Blattanalysen durchwegs im Normalbereich zwischen 1 und $1.4 \%$ Kalium in der Trockensubstanz. Dies ohne zwischenzeitlicher Düngung, allein infolge der höheren Bodenfeuchtigkeit im Jahre 2016. Weiterhin zeigen die Blattanalysen den Antagonismus zwischen der Kalium- und Magnesiumaufnahme klar auf. Die beiden Anlagen mit dem höchsten Kaliumgehalt weisen in beiden Jahren die niedrigsten Magnesiumgehalte auf. Besonders bei der Anlage „Burg“ lagen die MagnesiumBlattgehalte weitgehend im Mangelbereich und diese Anlage wies auch als einzige der sechs untersuchten einen Befall durch Stiellähme und Grüne Beeren auf.

2016 wurden zu zwei Terminen Bodenproben für Analysen entnommen. Die Ergebnisse waren diesmal an beiden Terminen ähnlich, variierten jedenfalls weniger als im Vorjahr (Abb. 2).

\section{KALIUM IM MOST, PH-WERTE UND WEINSÄURE}

Im Laufe der Reifeentwicklung stieg der Kaliumgehalt in den Mosten erwartungsgemäß an. Zur Ernte schwankten die Kaliumwerte der Moste aus den sechs Anlagen zwischen 1.5 und etwas über $2 \mathrm{~g} / \mathrm{l}$ Most (Abb. 4 und Abb. 5). Bereits im Laufe der Gärung und weiter auch während der Weinsteinstabilisierung fiel Kalium aus, und zwar umso mehr, je mehr Kalium im Most zur Ernte enthalten war. Dabei bindet sich das Kalium an die Weinsäure und es fällt somit Weinsäure aus. Die Abbildung 4 zeigt, dass die Moste mit den niedrigsten Kaliumgehalten zur Ernte schließlich die höchsten Weinsäuregehalte aufwiesen, etwa doppelt so viel wie die Weine aus den Anlagen mit dem höchsten Kaliumgehalt. Allerdings lagen die Weinsäurewerte insgesamt auf eher niedrigem Niveau. Die pH-Werte der Versuchsweine schwankten zwischen 3.44 und 3.58, wobei die Anlagen „Berg“ und „Tal“ die niedrigsten Werte aufwiesen. Die pH-Werte der restlichen vier Anlagen lagen sehr nahe beisammen. Im trockenen Jahr 2015 waren die Erträge durchwegs gering, unter der angestrebten Menge von $80 \mathrm{dt} / \mathrm{ha}$, nur die Anlagen "Berg" und „Tal“ wiesen Erträge von über $100 \mathrm{dt} / \mathrm{ha}$ auf.

2016 waren ähnliche Ergebnisse zu verzeichnen. Obwohl die Proben bewusst zügig verarbeitet wurden, dürfte es zum Teil beim
Pressen zu einem vorzeitigen Weinsteinausfall gekommen sein. Zumindest deutet der starke Abfall der Weinsäure bei der Anlage „Burg“ im Most zur Ernte darauf hin (Abb. 5). Wiederum wiesen die Anlagen "Berg" und „Tal“ die niedrigsten Kaliumgehalte im Most zur Ernte auf, obwohl diesmal die Erträge angeglichen wurden. Nur bei der Anlage Tal waren zwei nicht ertragsreduzierte Parzellen belassen worden. Diese Variante mit dem höchsten Ertrag wies den mit Abstand niedrigsten $\mathrm{pH}$-Wert auf.

\section{WELCHE PARAMETER BEEINFLUS-} SEN DIE PH-WERTE DER VER-

\section{SUCHSWEINE?}

In Tabelle 1 sind die im Versuchszusammenhang relevanten Parameter angeführt, welche signifikant mit dem $\mathrm{pH}$-Wert der fertigen Weine korrelierten. Der pH-Wert im Most zur Ernte korrelierte signifikant mit dem $\mathrm{pH}$-Wert der fertigen Weine. Weiters standen, wie zu erwarten, die Weinsäure im Wein, die titrierbare Gesamtsäure und der Kaliumgehalt, enzymatisch oder auch mit FOSS gemessen, in einem signifikanten $\mathrm{Zu}$ sammenhang mit dem $\mathrm{pH}$-Wert der Weine. 2015 wiesen die Varianten mit den höheren Erträgen etwas niedrigere Zuckergehalte und zugleich etwas niedrigere Kaliumgehalte auf. Im Jahre 2016 hingegen lagen die Zuckerwerte der Moste sehr nahe beisammen. Daher erklärt sich die Korrelation zwischen dem Alkoholgehalt und dem $\mathrm{pH}$-Wert der Weine im Jahre 2015. Die Ertragsmengen wirkten sich in beiden Jahren sehr deutlich auf den $\mathrm{pH}$-Wert der Weine aus. Hingegen standen weder Blatt- noch Bodenkaliumgehalte in einem signifikanten Zusammenhang mit den $\mathrm{pH}$-Werten der Weine.

\section{DISKUSSION}

Obwohl die Kaliumgehalte im Blatt im Jahre 2015 sehr niedrig und durchwegs unter der Schwelle für eine ausreichende Versorgung (1\% Kalium in der Trockensubstanz) lagen, war eine hohe Einlagerung von Kalium in die Beeren zu verzeichnen. In beiden Jahren des Versuches lagen die niedrigsten Kaliumwerte im Most zur Ernte bei etwa $1.5 \mathrm{~g} / \mathrm{l}$. Vergleichsweise war der Wert im Versuch von Ryser et.al. 1989 [3] in der über Jahren nicht gedüngten Parzelle bei $1 \mathrm{~g} / \mathrm{l}$. Für hochwertige Burgunderweine wird von Kaliumgehalten im Most zur Ernte von $0.9 \mathrm{~g} / \mathrm{l}$ berichtet (ITV France, mündliche Mitteilung). Vermutlich wäre auch im vorliegenden Versuch, im teilweise sehr trockenen Jahr 2015 die Kaliumaufnahme in der Reifephase niedriger geblieben, hätte die Trockenphase bis über die Ernte angedauert. Hingegen genügten die Niederschläge im August und September 2015, um das Defizit in der Kaliumaufnahme auszugleichen.

Es stellt sich die Frage, ob es auch in Südtirol sinnvoll wäre, die Kaliumversorgung der Anlagen weiter abzusenken. Niedrigere Kaliumgehalte im Most zur Ernte würden einen geringeren Weinsteinausfall bewirken und höhere Weinsäuregehalte in den Weinen bewahren. Die Anreicherung der Moste und Weine mit Säure könnte damit vermieden oder minimiert werden. Wieweit dies vorteilhaft für die Qualität und die Entwicklung der Weine im Laufe ihrer Alterung wäre, ist nicht bekannt und wird konträr bewertet. Ob dies praktisch möglich und empfehlenswert wäre, bleibt weiterhin auszuloten. Es könnte mit einem höheren Risiko für akut auftretende Mangelsituationen in anhaltenden Trockenphasen verbunden sein. Allerdings sind im Südtiroler Weinbau Kaliummangelsituationen bisher so gut wie unbekannt, da die vorliegenden, durchwegs leichten, sandig-lehmigen Böden das Kalium leicht abgeben und nicht fixieren.

Während der Stickstoffgehalt der Blätter zu Reifebeginn durchwegs gut mit den hefeverwertbaren Stickstoffgehalten im Most korreliert (Linsenmeier et al., 2008) [7], war dieser Zusammenhang in vorliegender Arbeit für Kalium nicht feststellbar. Es konnte kein Zusammenhang zwischen den Kaliumgehalten im Blatt und jenen im Most gefunden werden. Dies dürfte wohl darauf hindeuten, dass Kalium noch in der Reifephase in beachtlichen Mengen aus dem Boden aufgenommen werden kann und dass das in den Blättern zu Reifebeginn enthaltene Kalium nur ein Teil des Kaliums ist, welches bei der Ernte im Most vorhanden ist. Es war im vorliegenden Versuch auch so, dass die Kaliumgehalte in den Blättern während der Reifephase nur in einem Fall leicht absanken, in allen anderen Anlagen aber gleichblieben oder vielfach auch leicht anstiegen. Auch die Kaliumgehalte der Böden korrelierten nicht mit jenen der Moste und Weine. Dies wäre auch kaum zu erwarten gewesen, da die Böden der untersuchten Standorte unterschiedlichen Bodenarten, vom lehmigen Sand bis zum schluffigen Lehm, zuzuordnen waren.

Laut den vorliegenden Ergebnissen scheint sich die Ertragshöhe auf die Kaliumgehalte der Moste auszuwirken. Höhere Erträge führten in beiden Untersuchungsjahren zu 
niedrigeren Kaliumgehalten im Most. Dieser Zusammenhang wurde auch von anderen Autoren gefunden (Hepner und Bravdo, 1985) [6], scheint aber nicht regelmäßig aufzutreten (Freeman and Kliewer) [8)]. Die laut Aussagen von Kellermeistern hohen $\mathrm{pH}$ Werte der Südtiroler Moste, könnten daher, zumindest zum Teil, auch auf die niedrigen Erträge vieler Anlagen zurückzuführen sein.

Überraschend bei den erhobenen KaliumWerten im Boden waren ihre beachtlichen Schwankungen zwischen den einzelnen Untersuchungsterminen. Besonders auffällig war dies bei den beiden Anlagen „Berg“ und „Tal“ im Jahre 2015. Diese beiden Anlagen wiesen im Frühjahr 2015 eine Winterbegrünung auf. Da auch bei den ersten Blattproben zur Blüte 2015 diese beiden Anlagen deutlich höhere Kaliumgehalte zeigten als in der Folge, stellt sich die Frage, ob und wieweit die Bodenbewirtschaftung den Kaliumhaushalt und die Kaliumaufnahme beeinflusst. Der Umstand, dass in Südtirol, trotz sehr reduzierter Kaliumdüngung, nach wie vor bei Blatt- und Bodenanalysen in vielen Fällen hohe Kaliumgehalte gefunden werden, wird zum Teil auf die flächendeckend verbreitete Dauerbegrünung zurückgeführt. Diese soll dazu beitragen, das Kalium im Oberboden zu halten und anzureichern. Von einzelnen Beratern wird daher eine Umstellung in der Bodenbewirtschaftung angeregt, um mittelfristig zu niedrigeren Kaliumgehalten in den Mosten zu kommen. Wieweit dies machbar ist, bedarf weiterer Abklärung.

Schließlich haben die hier vorliegenden Ergebnisse klar aufgezeigt, dass einzelne Blattanalysen keine verlässliche Information über einen gegebenen Düngungsbedarf bei Kalium erlauben. Dazu hängen die Kaliumgehalte der Blätter zu stark von den vorherrschenden klimatischen Gegebenheiten ab. Besonders anhaltende Trockenphasen schränken die Kaliumaufnahme seitens der Rebe stark ein und lassen die Kalium-Blattwerte deutlich in den Mangelbereich absinken. Mangelsymptome an Reben oder Anzeichen von negativen Auswirkungen derartiger Phasen konnten bisher nicht festgestellt werden. Allerdings ist es meist so, dass sich in Südtirol Trockenphasen bisher so gut wie nie über eine ganze Vegetationsperiode erstreckten, sondern immer wieder durch kürzere oder längere Niederschlagsphasen oder zumindest durch Sommergewitter unterbrochen wurden. Bei Anlagen, die über viele Jahre nicht mit Kalium gedüngt wurden, ist eine sich anbahnende Unterversorgung, welche in extremen Trockenphasen

\section{ZUSAMMENFASSUNG}

Die pH-Werte der Südtiroler Moste und Weine sind in der Mehrzahl der Jahre hoch. Die hohen pH-Werte hängen nicht nur mit den niedrigen Säurewerten zur Reife zusammen, sie sind auch die Folge hoher Kaliumgehalte im Most. Es sollten daher erste Erkenntnisse gewonnen werden, wieweit Rebanlagen mit niedrigen Kaliumgehalten laut Blattanalyse auch Moste mit niedrigeren $\mathrm{pH}$-Werten ergeben und ob es angezeigt wäre, ein größeres Augenmerk auf eine noch gezieltere und evtl. auch noch niedrigere Kaliumversorgung der Reben zu legen.

Zu diesem Zwecke wurden drei Anlagen, welche in den letzten Jahren wiederholt niedrige Kalium-Blattwerte aufwiesen, und drei höher versorgte Anlagen bezüglich Kaliumgehalt im Boden, im Blatt und in den Mosten bis hin zum fertigen Wein untersucht.

Es zeigte sich, dass alle Anlagen, auch die mäßig versorgten, immer noch zu hohe Kaliumgehalte in den Böden aufwiesen, um deutlich niedrigere Kaliumgehalte in den Mosten zu bewirken. Selbst in Jahren mit sehr niedrigen Kaliumwerten im Blatt zur Blüte und zu Reifebeginn, wurden in den Mosten immer noch 1.5-2.1 g/I Kalium gefunden. In ausgeprägten Trockenphasen, wie sie in den letzten Jahren infolge der Klimaänderung verstärkt auftraten, ist die Kaliumaufnahme durch die Rebe zwar stark eingeschränkt, solange die Trockenheit anhält, die Defizite können aber rasch ausgeglichen werden, sobald höhere Bodenfeuchte vorliegt.

\section{RIASSUNTO}

Per la maggior parte delle annate, il valore di $\mathrm{pH}$ dei mosti e vini dell'Alto Adige risulta elevato. Questo fenomeno non è correlato soltanto alla ridotta acidità alla vendemmia, ma dipende anche dall'alto tenore di potassio nei mosti. Si è voluto perciò verificare se e in quale misura il limitato assorbimento di potassio possa influenzare il pH dei mosti e dei vini e se sia opportuno considerare una somministrazione inferiore ed ancora più mirata di potassio.

A questo scopo sono stati studiati tre vigneti che negli ultimi anni hanno ripetutamente evidenziato bassi valori di potassio nelle analisi fogliari e tre vigneti meglio approvvigionati, rilevando il contenuto di potassio nel suolo, nelle foglie, nei mosti e nei vini.

I risultati confermano che in tutti i vigneti, anche in quelli con minor dotazione, il terreno mostra contenuti di potassio troppo elevati per influenzare negativamente il dato relativo ai mosti. Anche in annate nelle quali le analisi fogliari indicavano valori di potassio molto bassi in fioritura e all'invaiatura sono stati riscontrati nei mosti 1,5-2,1 g/l di potassio. In occasione di lunghi periodi siccitosi, frequenti negli ultimi anni a causa del cambiamento climatico, l'assorbimento di potassio da parte della vite risulta ridotto finché la siccità persiste, ma il deficit viene rapidamente compensato con l'incremento dell'umidità nel terreno.

akut werden könnte, jedenfalls im Auge zu behalten.

\section{SCHLUSSFOLGERUNGEN}

Drei Standorte mit mäßiger und drei mit höherer Kaliumversorgung wurden über zwei Jahre näher untersucht. Es zeigte sich, dass 
die Kaliumblattwerte in Trockenphasen deutlich in den Mangelbereich abfallen können, auch bei gut mit Kalium versorgten Anlagen. Negative Auswirkungen oder Mangelerscheinungen sind infolge der niedrigen Kalium-Blattwerte nicht aufgetreten. Die Niederschläge im Sommer und Frühherbst reichten aber selbst im anfänglich sehr trockenen Jahr 2015 aus, um in allen Anlagen,

\section{LITERATUR}

[1] Adler S., Chimani B., Drechsel S., Haslinger K., Hiebl J., Meyer V., Resch G., Rudolph J., Vergeiner J., Zingerle Ch., Marigo G., Fischer A., Seiser B. (2015). Das Klima von Tirol - Südtirol - Belluno. Zentralanstalt für Meteorologie und Geodynamik (ZAMG), [Wien].

[2] Boulton R. (1980). The general relationship between potassium, sodium and $\mathrm{pH}$ in grape juice and wine. American Journal of Enology and Viticulture 31 (2), 182-186.

[3] Ryser J.P., Aerny J., und Murisier F. (1989). Fumure potassique de la vigne e aciditè du vin. Revue suisse de Viticulture, Arboriculture, Horticulture 21 (5), 319-323. unabhängig von der Verfügbarkeit im Boden, Kaliumgehalte von 1.5 bis etwa $2.1 \mathrm{~g} / \mathrm{l}$ im Most zu erreichen. In Qualitätsweinbaugebieten werden heute auch niedrigere Kaliumgehalte angestrebt. Diese würden zu einem geringeren Ausfall an Kalium mit Weinsäure und zu höheren Säuregehalten und niedrigeren $\mathrm{pH}$-Werten im Wein führen. Um

[4] Schneider V. (1998). Kalium. Sensorische Bedeutung und önologische Differenzierung. Die Winzer-Zeitschrift (7), 3639.

[5] Keller M. (2015). The Science of Grapevines. Anatomy and physiology. Academic Press, Amsterdam, hier chapter “Potassium", pp. 300-303.

[6] Hepner Y., Bravdo B. (1985). Effect of Crop Level and Drip Irrigation Scheduling on the Potassium Status of Cabernet Sauvignon and Carignane Vines and Its Influence on Must and Wine Composition and Quality. American Journal of Enology and Viticulture 36 (2), 140-147. dieses Ziel zu erreichen, müsste die Kaliumversorgung der Südtiroler Weinbauböden weiter abgesenkt werden. Wieweit dies möglich und sinnvoll ist, bleibt weiter auszuloten.

[7] Linsenmeier A., Loos U., Löhnertz O. (2008). Must composition and nitrogen uptake in a long-term trial as affected by timing of nitrogen fertilization in a cool-climate Riesling vineyard. American Journal of Enology and Viticulture 59 (3), 255-264.

[8] Freeman B.M. and Kliewer, W.M. (1983). Effect of irrigation, crop level and potassium fertilization on Carignane vines II. Grape and wine quality. American Journal of Enology and Viticulture 34, 197-207. 


\section{ANHANG 1: TABELLEN}

Tab. 1: Parameter, die 2015 und 2016 eine signifikante Korrelation mit dem pH-Wert der Weine aufwiesen. // Parameters with a significant correlation on the $\mathrm{pH}$ values of the wines in the years 2015 and 2016.

\begin{tabular}{|c|c|c|c|}
\hline \multicolumn{2}{|c|}{ Korrelationen } & \multirow{2}{*}{$\begin{array}{c}2015 \\
\text { pH-Wert im Wein } \\
.818^{*}\end{array}$} & \multirow{2}{*}{$\begin{array}{c}2016 \\
\text { pH-Wert im Wein } \\
.886^{*}\end{array}$} \\
\hline \multirow{3}{*}{ pH-Wert zur Ernte } & Pearson Correlation & & \\
\hline & Sig. (2-tailed) & 0.001 & 0 \\
\hline & $\mathrm{N}$ & 12 & 19 \\
\hline \multirow{3}{*}{ tit_Gesamtsäure im Wein } & Pearson Correlation & $-.742^{*}$ & $-.852^{*}$ \\
\hline & Sig. (2-tailed) & 0.006 & 0 \\
\hline & $\mathrm{N}$ & 12 & 19 \\
\hline \multirow{3}{*}{ Weinsäure im Wein } & Pearson Correlation & $-.864^{*}$ & $-.815^{*}$ \\
\hline & Sig. (2-tailed) & 0.000 & 0 \\
\hline & $\mathrm{N}$ & 12 & 19 \\
\hline \multirow{3}{*}{ Alkohol } & Pearson Correlation & $.851^{*}$ & 0.131 \\
\hline & Sig. (2-tailed) & 0.000 & 0.594 \\
\hline & $\mathrm{N}$ & 12 & 19 \\
\hline \multirow{3}{*}{ Kalium im Wein (enzymatisch) } & Pearson Correlation & b. & $.905^{*}$ \\
\hline & Sig. (2-tailed) & & 0 \\
\hline & $\mathrm{N}$ & 0 & 19 \\
\hline \multirow{3}{*}{ Kalium im Wein (FOSS) } & Pearson Correlation & $.825^{*}$ & $.898^{*}$ \\
\hline & Sig. (2-tailed) & 0.001 & 0 \\
\hline & $\mathrm{N}$ & 12 & 19 \\
\hline Trauben kg/qm & Pearson Correlation & $-.815^{*}$ & $-.849 *$ \\
\hline
\end{tabular}




\section{ANHANG 2: ABBILDUNGEN}

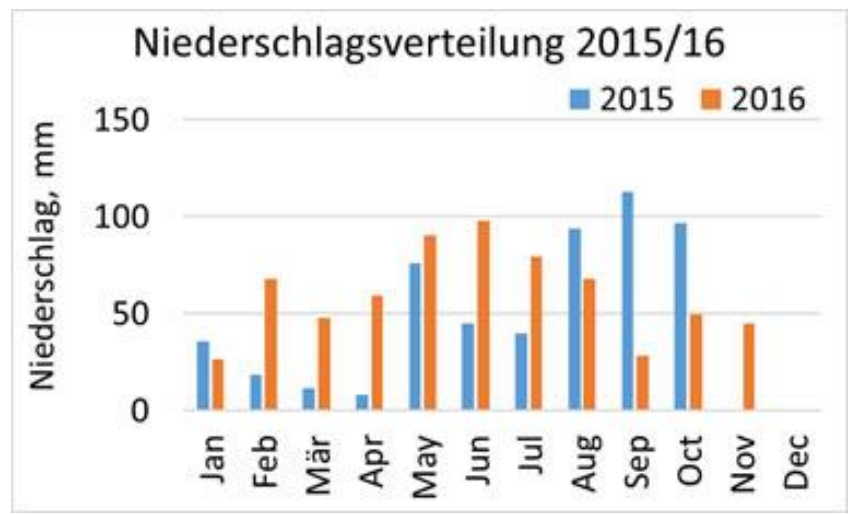

Abb. 1: Niederschlagsverteilung in den Jahren 2015 und 2016, laut Wetterstation Laimburg. // Rainfall distribution in the years 2015 and 2016 , recorded from the meteorological station at Laimburg.

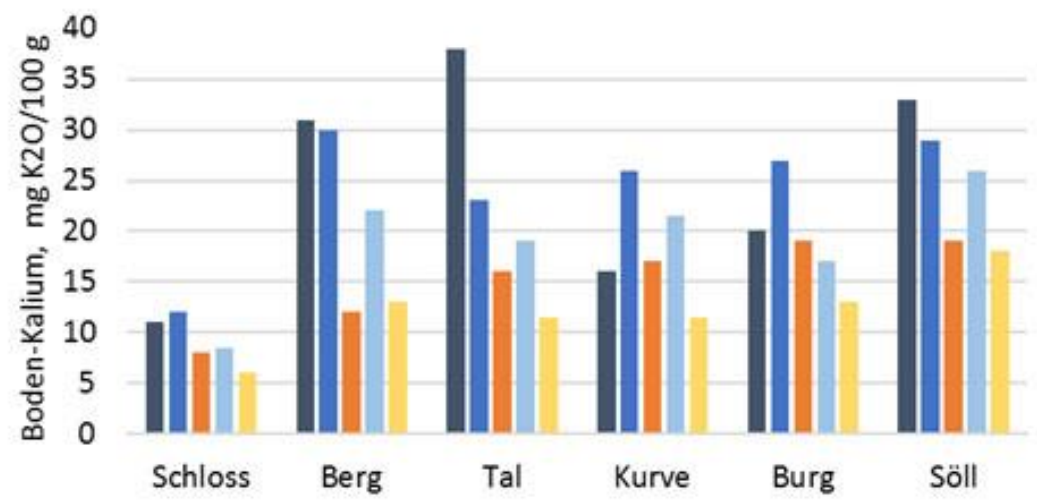

@ Oberb. 24.04.2015 Oberb. 18.08.2015 = Unterb. 18.08.2015

in Oberb. 24.09.2015 $=$ Unterb. 24.09.2015

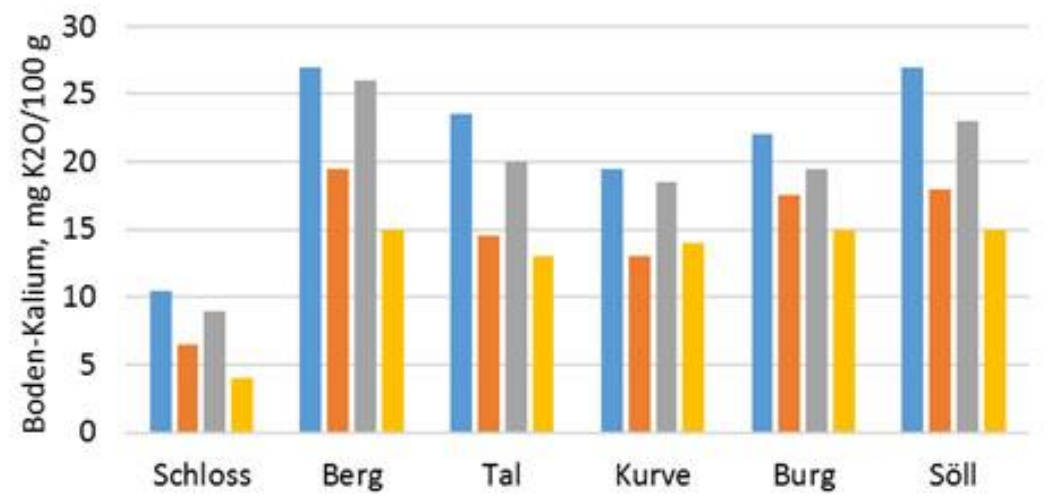

= Oberb. 24.05.2016 = Unterb. 24.05.2016

$=$ Oberb. 01.08.2016 $=$ Unterb. 01.08.2016

Abb. 2: Kaliumgehalte der Versuchsanlagen laut Bodenanalysen in den Versuchsjahren 2015 und 2016. // Potassium contents of the experimental sites in the soil analyses of the trial years 2015 and 2016. 

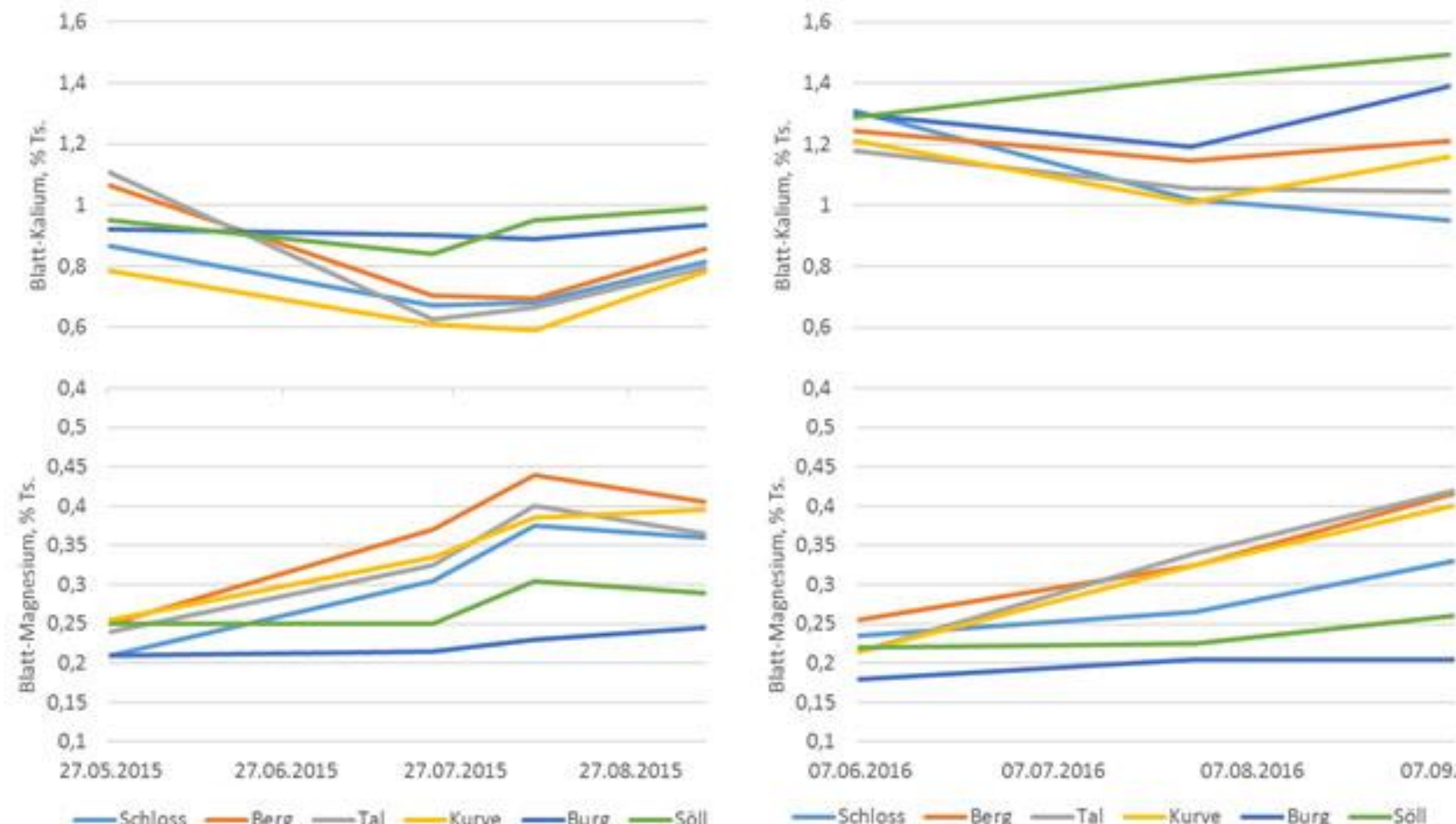

$$
0,6
$$

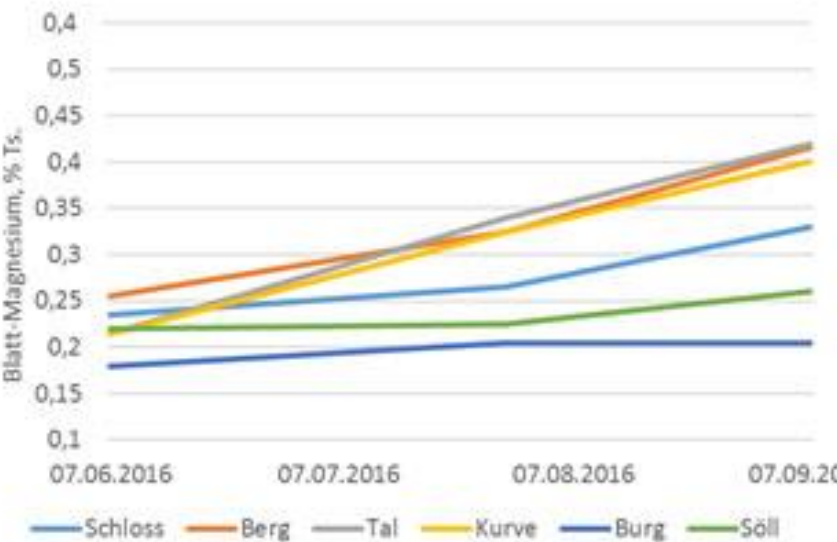

Abb. 3: Kalium- und Magnesiumgehalte in den Blättern der Versuchsanlagen in den beiden Untersuchungsjahren. // Potassium and Magnesium contents in the leaves of the experimental vineyards in the two years of the investigation. 


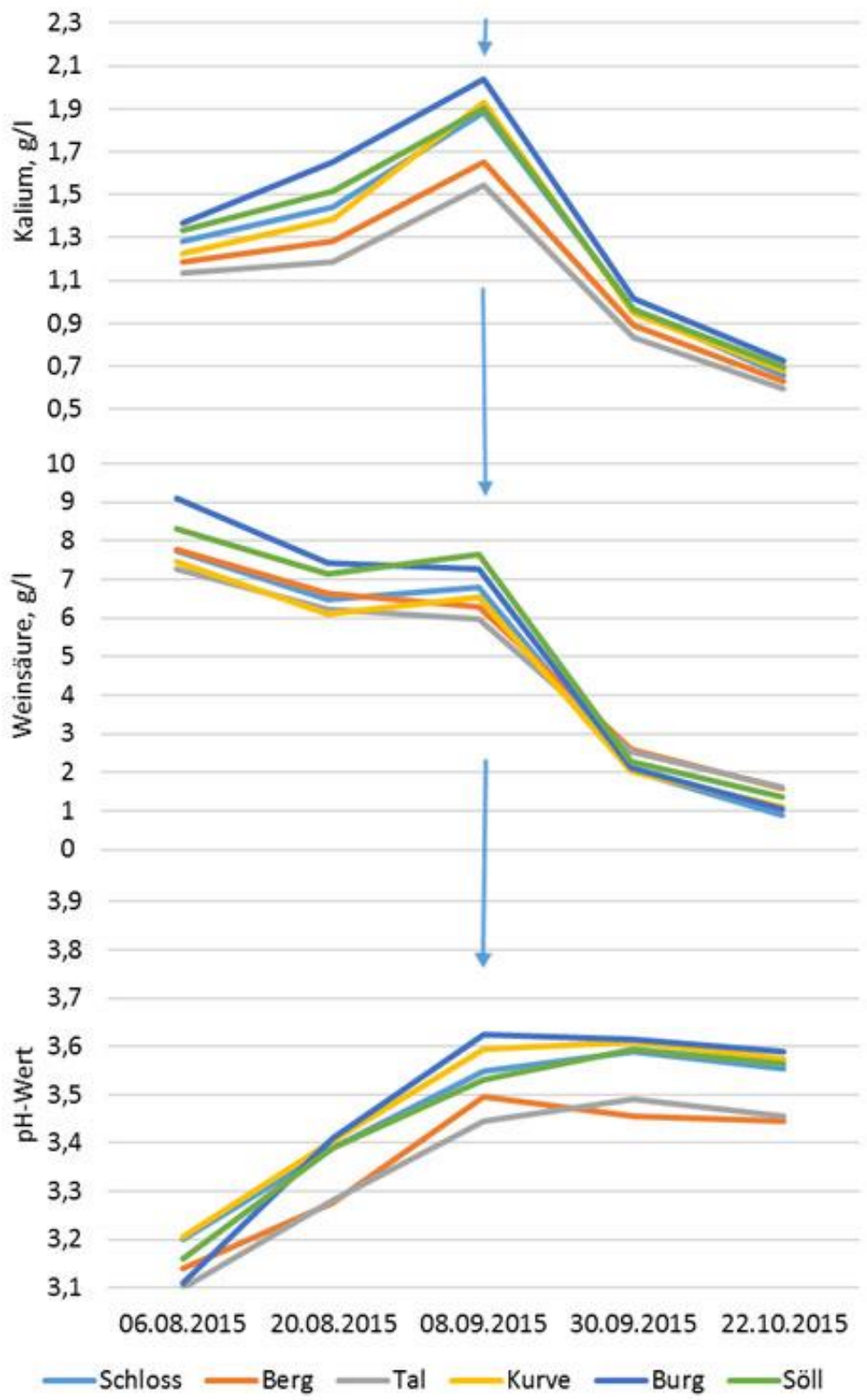

Abb. 4: Entwicklung der Kaliumgehalte, Weinsäure und pH-Werte der Moste und Weine der verschiedenen Versuchsanlagen im Jahre 2015 (die Pfeile kennzeichnen den Erntezeitpunkt). // Development of the potassium levels, tartaric acid and pH values of the musts and wines of the different experimental vineyards in 2015 (the arrows indicate the date of harvest). 

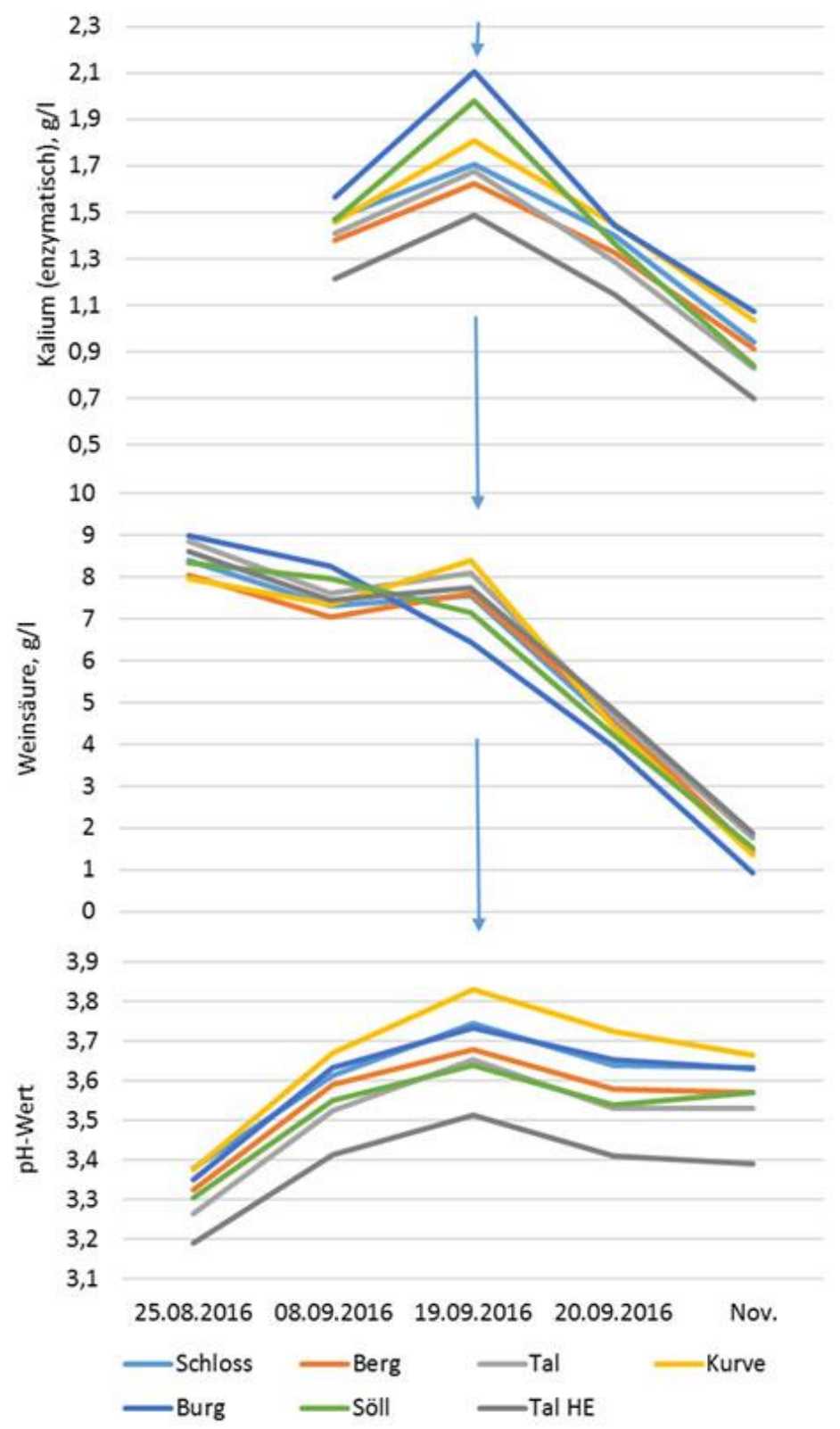

Abb. 5: Entwicklung der Kaliumgehalte, Weinsäure und pH-Werte der Moste und Weine der verschiedenen Versuchsanlagen im Jahre 2016 (die Pfeile kennzeichnen den Erntezeitpunkt). // Development of the potassium levels, tartaric acid and pH values of the musts and wines of the different experimental vineyards in 2016 (the arrows indicate the date of harvest).

\section{(c) (1) @}

Dieses Werk ist lizenziert unter einer Creative Commons Namensnennung - Nicht kommerziell 4.0 International Lizenz.

Quest'opera è distribuita con Licenza Creative Commons Attribuzione - Non commerciale 4.0 Internazionale.

This work is licensed under a Creative Commons Attribution - NonCommercial 4.0 International License.

Für alle Abbildungen und Tabellen ohne Nennung des Urhebers gilt: @ Versuchszentrum Laimburg.

Per tutte le immagini e tabelle senza menzione dell'artefice vale: (c) Centro di Sperimentazione Laimburg.

For all figures and tables without mention of the originator applies: (C) Laimburg Research Centre. 